\title{
Wert und Preis
}

\section{Samia Hurst}

Prof. Dr. med., Institut Ethique Histoire Humanités (iEH2), Faculté de Médecine, Genève

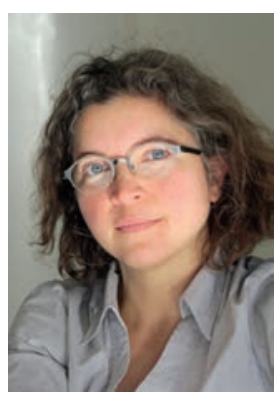

$\mathrm{Zu}$ welchen Opfern sind wir bereit, wenn wir nicht immer noch mehr bezahlen wollen? Diese Frage ist leider mit der Grenfell-Brandkatastrophe in London am 14. Juni erschreckend aktuell geworden. Das Feuer, das fast 80 Todesopfer forderte, sei teilweise durch eine Gebäudeverkleidung beschleunigt worden, die bekanntlich feuergefährlich ist. Sie sei leicht günstiger als eine Gebäudeverkleidung der Konkurrenz. Wirklich leicht günstiger: Die Ersparnis für das gesamte Gebäude hätte ungefähr $£ 5000$ betragen. Man versteht die Wut der Überlebenden.

Geld sparen, grundsätzlich eine gute Sache, kann problematisch werden, wenn man dabei Dinge opfert, die die Zusatzkosten mehr als wert gemacht hätten. Diese kleine - eigentlich banale - Feststellung hätte tiefgreifende Folgen gehabt, wenn wir sie wirklich ernst genommen hätten. Denn was wir beim Geldsparen opfern, ist oft unsichtbar. Und obwohl die Bewohner des Grenfell Tower für die Besitzer hätten sichtbar sein müssen, passiert es doch, dass diese Unsichtbarkeit naheliegender ist. $\mathrm{Zu}$ diesem Thema hat meine Kollegin Annette Rid kürzlich einen sehr interessanten Artikel über die Budget-Wirkungsanalyse verfasst [1]. Erinnern Sie sich noch an den Beschluss über die

Geld sparen, grundsätzlich eine gute Sache, kann problematisch werden, wenn man dabei Dinge opfert, die die Zusatzkosten mehr als wert gemacht hätten.

Kostenerstattung von Sofosbuvir gegen Hepatitis C? Dies ist ein eindeutig wirksames Medikament, dessen Kosten-Nutzen-Analyse zeigt, dass das Preis-/Qualitätsverhältnis innerhalb der in der Schweiz allgemein anerkannten Margen liegt [2]. Nur: Die Anzahl betroffener Patienten bewirkt, dass die Rechnung absolut untragbar wird. Deshalb werden die Kosten für das Medikament auch nur den am stärksten betroffenen Patienten zurückerstattet. Dies also ist die Budget-Wirkungsanalyse.

Im Gegensatz zur Brandkatastrophe von London scheint dieser Beschluss also erst einmal vernünftig.
Wenn wir nicht über die Mittel verfügen, dann ist es eben so. Das Kriterium der Priorität für die am meisten Betroffenen ist sogar gerecht: Sie sind es, die einen Unterschied am ehesten verspüren; sie sind es auch, die am wenigsten lange darauf warten können. Und dennoch: Auch hier opfern wir unsichtbare Dinge. Die Ablehnung einer Behandlung aufgrund der Anzahl betroffener Patienten führt zu einer krassen Ungleichbehandlung unter den Patienten: Einige werden weniger gut behandelt aus dem einfachen Grund, dass sie einer grösseren Patientengruppe angehören. Somit wird ein Teil der Gerechtigkeit geopfert. Die Ablehnung der Behandlung macht diese Patienten zu Geiseln in den Verhandlungen zwischen den Anbietern und den Staaten: Ein moralisches Problem, bei dem jede Seite zwar ihre Rolle spielt, aber trotzdem ein Problem. Ein Teil der Patientenrechte wird geopfert. Und wenn schliesslich der Zugang zu einer Behandlung mit hohen Kostenfolgen begrenzt wird, entfällt die Möglichkeit, die fehlenden Mittel dadurch zu kompeniseren, dass man auf weniger wirksame Leistungen verzichtet. Hier wird die Effizienz geopfert, ein Teil des Guten, das man mit den vorhandenen Mitteln bewirken könnte. Was also ist zu tun? Zurzeit gibt es keine Wunderrezepte. Könnten wir uns mit einem systematischeren Mechanismus ausstatten, um zu entscheiden, was wir aus einem Budget herausnehmen wollen, wenn eine solche Situation entsteht? Würden wir genauer hinschauen und unter Umständen sogar entscheiden, diese Opfer nicht zu erbringen? Es würde sicher nicht einfach sein, aber wenigstens hätten wir dann den Wert vor Augen und nicht nur den Preis.

\section{Literatur}

1 Charlton V, Littlejohns P, Kieslich K, Mitchell P, Rumbold B, Weale A, et al. Cost effective but unaffordable: an emerging challenge for health systems. Bmj. 2017 Mar 22;356:j1402. PubMed PMID: 28330879.

2 Pfeil AM, Reich O, Guerra IM, Cure S, Negro F, Mullhaupt B, et al. Cost-effectiveness analysis of sofosbuvir compared to current standard treatment in Swiss patients with chronic hepatitis C. PloS one. 2015;10(5):e0126984. PubMed PMID: 25974722. Pubmed Central PMCID: 4431849 